TRANSACTIONS OF THE

AMERICAN MATHEMATICAL SOCIETY

Volume 355, Number 1, Pages 49-55

S 0002-9947(02)03126-

Article electronically published on September 6, 2002

\title{
NONDEGENERATE MULTIDIMENSIONAL MATRICES AND INSTANTON BUNDLES
}

\author{
LAURA COSTA AND GIORGIO OTTAVIANI
}

\begin{abstract}
In this paper we prove that the moduli space of rank $2 n$ symplectic instanton bundles on $\mathbb{P}^{2 n+1}$, defined from the well-known monad condition, is affine. This result was not known even in the case $n=1$, where by Atiyah, Drinfeld, Hitchin, and Manin in 1978 the real instanton bundles correspond to self-dual Yang Mills $S p(1)$-connections over the 4-dimensional sphere. The result is proved as a consequence of the existence of an invariant of the multidimensional matrices representing the instanton bundles.
\end{abstract}

\section{INTRODUCTION}

A symplectic instanton bundle on $\mathbb{P}_{\mathbb{C}}^{2 n+1}$ is a bundle of rank $2 n$ defined as the cohomology bundle of a well-known monad (see Definition 2.2.).

In ADHM78, it was shown that instanton bundles on $\mathbb{P}^{3}$ satisfying a reality condition correspond to self-dual Yang Mills $S p(1)$-connections over the 4-dimensional sphere $S^{4}=\mathbb{P}_{\mathbb{H}}^{1}$. This correspondence was generalized by Salamon ([Sal84]) who showed that instanton bundles on $\mathbb{P}^{2 n+1}$ which are trivial on the fiber of the twistor map $\mathbb{P}^{2 n+1} \rightarrow \mathbb{P}_{\mathbb{H}}^{n}$ correspond to $S p(n)$-connections which minimize a certain Yang Mills functional over $\mathbb{P}_{\mathbb{H}}^{n}$. We denote by $M I_{\mathbb{P}^{2 n+1}}(k)$ the moduli space of symplectic instanton bundles on $\mathbb{P}^{2 n+1}$ with $c_{2}=k$ (see Definition 2.4) and we denote by $I_{\mathbb{P}^{2 n+1}}(k)$ the moduli space of $k$-instanton bundles on $\mathbb{P}^{2 n+1}$ (see Definition 4.1).

Up to now, very little is known concerning the geometry of the moduli spaces $I_{\mathbb{P}^{2 n+1}}(k)$ and a few results have been proved regarding $M I_{\mathbb{P}^{2 n+1}}(k)$. For instance, up to the authors' knowledge, the only results concerning $M I_{\mathbb{P}^{2 n+1}}(k)$ deal with small values of $n$ and $k$. Indeed, it is known (ADHM78]) that $M I_{\mathbb{P}^{2 n+1}}(k)$ has a component of dimension $8 k-3$ for $n=1$, that it is smooth for $n=1$ and $k \leq 5$ (KO99]) but, it is conjectured that it is singular and reducible for $n \geq 2$ and $k \geq 4$ (see [AO00]).

The goal of this paper is to show that all the moduli spaces $M I_{\mathbb{P}^{2 n+1}}(k)$, for any $n \geq 1$ and any $k \geq 1$, share the following surprising property:

Theorem 1.1. $M I_{\mathbb{P}^{2 n+1}}(k)$ is affine.

In addition, we will see that the same holds for all moduli spaces parametrizing $k$-instanton bundles on $\mathbb{P}^{2 n+1}$. Indeed, we will prove

Theorem 1.2. $I_{\mathbb{P}^{2 n+1}}(k)$ is affine.

Received by the editors October 23, 2001.

2000 Mathematics Subject Classification. Primary 14D21, 14J60; Secondary 15A72.

The first author was partially supported by DGICYT BFM2001-3584.

The second author was partially supported by Italian MURST.

(C)2002 American Mathematical Society 
As a by-product of Theorems 1.1 and 1.2, we will contribute to the study of a problem posed in the 80 's (see for instance [HH86]) that, in the context of instanton bundles on $\mathbb{P}^{2 n+1}$, reads as follows:

Problem. Determine the maximal dimension of complete subvarieties lying on $M I_{\mathbb{P}^{2 n+1}}(k)\left(\right.$ resp. $\left.I_{\mathbb{P}^{2 n+1}}(k)\right)$.

More precisely, in this case, we will completely solve the problem and in Corollaries 3.5 and 4.6 we will see that $M I_{\mathbb{P}^{2 n+1}}(k)$ (resp. $\left.I_{\mathbb{P}^{2 n+1}}(k)\right)$ does not contain any complete subvariety of positive dimension.

The technique we use to prove our main results is to exhibit $M I_{\mathbb{P}^{2 n+1}}(k)$ (resp. $\left.I_{\mathbb{P}^{2 n+1}}(k)\right)$ as the GIT-quotient of an affine variety $\mathcal{Q}^{0}$ (resp. $\mathcal{P}^{0}$ ) and then use standard results in invariant theory. The fact that $\mathcal{Q}^{0}$ (resp. $\mathcal{P}^{0}$ ) is affine is a consequence of the existence of an invariant of multidimensional matrices representing the instanton bundles, which generalizes the hyperdeterminant (see GKZ94 and AO99]).

The first named author would like to thank the Dipartimento di Matematica, U. Dini for their hospitality and support at the time of the preparation of this paper.

\section{Notation AND PRELIMINARIES}

We will start by fixing some notation and recalling some facts about $k$-instanton bundles on $\mathbb{P}^{2 n+1}=\mathbb{P}(V)$, where $V$ is a complex vector space of dimension $2 n+2$. (See, for instance, OS86] and [AO94.)

Notation 2.1. $\mathcal{O}(d)=\mathcal{O}_{\mathbb{P}^{2 n+1}}(d)$ denotes the invertible sheaf of degree $d$ on $\mathbb{P}^{2 n+1}$ and for any coherent sheaf $E$ on $\mathbb{P}^{2 n+1}$ we denote $E(d)=E \otimes \mathcal{O}_{\mathbb{P}^{2 n+1}}(d)$.

Definition 2.2. A symplectic instanton bundle $E$ over $\mathbb{P}^{2 n+1}=\mathbb{P}(V)$ is a bundle of rank $2 n$ which appears as a cohomology bundle of a monad,

$$
I^{*} \otimes \mathcal{O}(-1) \stackrel{A}{\rightarrow} W \otimes \mathcal{O} \stackrel{A^{t}}{\rightarrow} I \otimes \mathcal{O}(1),
$$

where $(W, J)$ is a symplectic complex vector space of dimension $2 n+2 k$ and $I$ is a complex vector space of dimension $k$.

We do not assume in the definition that $E$ is stable, so we have to recall some results.

The monad condition means that $A$ is injective (as a bundle morphism), $A^{t}$ is surjective and $\operatorname{im} A \subset \operatorname{ker} A^{t}$ so that $E \simeq \operatorname{ker} A^{t} / \operatorname{im} A$. The fact that the map $W \otimes$

$\mathcal{O} \stackrel{A^{t}}{\longrightarrow} I \otimes \mathcal{O}(1)$ is surjective, is equivalent to the fact that the matrix $A \in \operatorname{Hom}\left(V^{*} \otimes\right.$ $I^{*}, W$ ) representing $E$ is nondegenerate according to [GKZ94] (see Definition 2.3 for the precise definition).

$\operatorname{Hom}\left(V^{*} \otimes I^{*}, W\right)$ contains the subvariety $\mathcal{Q}$ given by matrices $A$ for which the sequence (11) is a complex, that is, such that $A^{t} J A=0 . G L(I) \times S p(W)$ acts on $\mathcal{Q}$ by $(g, s) \cdot A=s A g$.

Definition 2.3. A matrix $A \in \operatorname{Hom}\left(V^{*} \otimes I^{*}, W\right)$ is called degenerate if the multilinear system $A(v \otimes i)=0$ has a solution such that $0 \neq v \in V^{*}$ and $0 \neq i \in I^{*}$.

By [GKZ94, Theorem 14.3.1, this is equivalent to the standard definition of degeneracy given in chapter 14.1 of [GKZ94. It is easy to check that degenerate matrices fill an irreducible subvariety $N$ of $\operatorname{Hom}\left(V^{*} \otimes I^{*}, W\right)$ of codimension $k$ (see [WZ96]). Hence, only in the case $k=1$ is it well-defined as a hyperdeterminant 
according to GKZ94]. In the next section we will define an $S L(I) \times S p(W)$ invariant on $\operatorname{Hom}\left(V^{*} \otimes I^{*}, W\right)$, called $D$, which generalizes the hyperdeterminant and is suitable for our purposes.

It was shown in AO94 that all instanton bundles are simple, so that they carry a unique symplectic form. Moreover, for $n=1,2$ it was proved in A094 that all instanton bundles are stable, and it is expected that the same result is true for $n \geq 3$.

Recall that given $X=\operatorname{Spec}(A)$, an affine scheme, and a reductive group $G$ acting on $X$, then a theorem of Hilbert and Nagata shows that the ring of invariants $A^{G}$ is finitely generated and $X / G:=\operatorname{Spec}\left(A^{G}\right)$ is what is called the affine algebrogeometric quotient of $X$ by $G$. In addition, $X / G$ is a good quotient and it is a geometric quotient if and only if all orbits are closed. In this setting, every orbit contains a unique closed orbit in its closure and a point in $X$ is called stable if its orbit is closed and has the maximal dimension (see [PV89]).

In [BH78] it was essentially proved that there is a natural one-to-one correspondence between

i) isomorphism classes of symplectic instanton bundles, and

ii) orbits of $G L(I) \times S p(W)$ on the open subvariety $\mathcal{Q}^{0}$ of $\mathcal{Q}$ given by nondegenerate matrices.

In fact, using the quoted results of AO94], one can see that BH78, Section 4 and the Theorem on page 19, adapt literally to our situation.

Moreover, in Theorem 3.3 we will see that $\mathcal{Q}^{0}$ is affine. Hence, if we denote by $G$ the quotient of $G L(I) \times S p(W)$ by $\pm(i d, i d)$, Barth and Hulek proved in BH78 that $G$ acts freely on $\mathcal{Q}^{0}$ and, in particular, all orbits are closed (in fact, any orbit contains in the closure orbits of smaller dimension). Therefore, all points of $\mathcal{Q}^{0}$ are stable for the action of $G L(I) \times S p(W)$ and $\mathcal{Q}^{0} \rightarrow \mathcal{Q}^{0} / G$ is a geometric quotient.

Definition 2.4. The GIT-quotient $\mathcal{Q}^{0} / G L(I) \times S p(W)$ is denoted by $M I_{\mathbb{P}^{2 n+1}}(k)$ and is called the moduli space of symplectic $k$-instanton bundles on $\mathbb{P}^{2 n+1}$. It is a geometric quotient.

The above discussion shows that $M I_{\mathbb{P}^{2 n+1}}(k)$ coincides for $n=1,2$ with the open subset $\mathcal{M I}_{\mathbb{P}^{2 n+1}}(k)$ of the Maruyama scheme of symplectic stable bundles on $\mathbb{P}^{2 n+1}$ of rank $2 n$ and Chern polynomial $\frac{1}{\left(1-t^{2}\right)^{k}}$ which are instanton bundles (this is an open condition because by Beilinson's theorem, it is equivalent to certain vanishing in cohomology; see [OS86]). In particular, our notation for $M I_{\mathbb{P}^{3}}(k)$ is consistent with the usual one. For $n \geq 3$ it is expected that the same result is true, but at present we can only say that $\mathcal{M I}_{\mathbb{P}^{2 n+1}}(k)$ is an open subset of $M I_{\mathbb{P}^{2 n+1}}(k)$.

\section{The invariant $D$ And the proof of the main RESUlt}

First, we remark that the vector spaces $W \otimes S^{n} I$ and $V \otimes S^{n+1} I$ have the same dimension $(2 n+2 k)\left(\begin{array}{c}k+n-1 \\ n\end{array}\right)=(2 n+2)\left(\begin{array}{l}k+n \\ n+1\end{array}\right)$. We can construct from

$$
W \stackrel{A^{t}}{\longrightarrow} V \otimes I
$$

the morphisms

$$
\begin{gathered}
A^{t} \otimes i d_{S^{n} I}: W \otimes S^{n} I \rightarrow V \otimes I \otimes S^{n} I, \\
i d_{V} \otimes \pi: V \otimes I \otimes S^{n} I \rightarrow V \otimes S^{n+1} I,
\end{gathered}
$$

where $\pi$ is the natural projection, and we consider the composition

$$
\Delta_{A}=\left(i d_{V} \otimes \pi\right) \cdot\left(A^{t} \otimes i d_{S^{n} I}\right): W \otimes S^{n} I \rightarrow V \otimes S^{n+1} I .
$$


Definition 3.1. Let $A \in \operatorname{Hom}\left(V^{*} \otimes I^{*}, W\right)$. We define $D(A)$ to be the usual determinant of the morphism $\Delta_{A}$ in (2) induced by $A$.

Notice that

$$
D: H \operatorname{Hom}\left(V^{*} \otimes I^{*}, W\right) \rightarrow(\operatorname{det} W)^{\alpha} \otimes(\operatorname{det} V)^{\beta}
$$

where $\alpha=-\left(\begin{array}{c}k+n-1 \\ n\end{array}\right)$ and $\beta=\left(\begin{array}{c}k+n \\ n+1\end{array}\right)$ is a $G L(V) \times G L(I) \times S p(W)$-equivariant map and $D(A)=0$ defines a homogeneous hypersurface of degree $(2 n+2 k)\left(\begin{array}{c}k+n-1 \\ n\end{array}\right)=$ $(2 n+2)\left(\begin{array}{c}k+n \\ n+1\end{array}\right)$. After a basis has been fixed in each of the vector spaces $V, I$ and $W$, the map $D$ can be seen as an $S L(V) \times S L(I) \times S p(W)$-invariant.

In fact, this definition generalizes the hyperdeterminant of boundary format as introduced in Theorem 14.3.3 of GKZ94.

Lemma 3.2. If $A$ is degenerate, then $D(A)=0$.

Proof. There are $0 \neq v \in V^{*}$ and $0 \neq i \in I^{*}$ such that $A(v \otimes i)=0$. Hence, $v \otimes S^{n+1} i \in V^{*} \otimes S^{n+1} I^{*}$ goes to zero under the dual of (2).

If $A$ is nondegenerate, we get $D(A) \neq 0$ only in the case $k=1$ and, in general, it can happen that $D(A)=0$, because the codimension of $N$ is $k$. Our main technical result is the following.

Theorem 3.3. If $A$ defines an instanton (that is, $A$ belongs to $\mathcal{Q}^{0}$ ), then $D(A) \neq 0$.

Proof. From (11) we get the exact sequence

$$
0 \rightarrow K \rightarrow W \otimes \mathcal{O} \rightarrow I \otimes \mathcal{O}(1) \rightarrow 0 .
$$

The $(n+1)$-th wedge power twisted by $\mathcal{O}(-n)$ gives the exact sequence

$$
\begin{aligned}
0 \rightarrow \wedge^{n+1} K(-n) & \rightarrow \wedge^{n+1} W(-n) \rightarrow \ldots \\
\ldots \rightarrow \wedge^{2} W \otimes S^{n-1} I(-1) & \rightarrow W \otimes S^{n} I \rightarrow S^{n+1} I(1) \rightarrow 0
\end{aligned}
$$

where the $H^{0}$ of the last morphism corresponds to $\Delta_{A}$ in (2). Taking cohomology, it is enough to prove

$$
H^{n}\left(\wedge^{n+1} K(-n)\right)=0 .
$$

The $(n+1)$-th wedge power twisted by $\mathcal{O}(-n)$ of the sequence

$$
0 \rightarrow I^{*} \otimes \mathcal{O}(-1) \rightarrow K \rightarrow E \rightarrow 0
$$

gives the sequence

$$
\begin{aligned}
0 & \rightarrow S^{n+1} I^{*} \otimes K(-2 n-1) \rightarrow \ldots \rightarrow \wedge^{n-1} K \otimes S^{2} I^{*}(-n-2) \rightarrow \ldots \\
& \ldots \rightarrow \wedge^{n} K \otimes I^{*}(-n-1) \rightarrow \wedge^{n+1} K(-n) \rightarrow \wedge^{n+1} E(-n) \rightarrow 0 .
\end{aligned}
$$

In order to prove (44), taking cohomology, we need $H^{n+i}\left(\wedge^{n-i} K(-n-i-1)\right)=0$ for $i=0, \ldots, n$ and $H^{n}\left(\wedge^{n+1} E(-n)\right)=0$. The first group of vanishing is easily obtained by taking suitable wedge powers of (3). The crucial point used to get the last vanishing is the isomorphism $\wedge^{n+1} E \simeq \wedge^{n-1} E$; it is true because $E$ is a rank $2 n$ vector bundle with $c_{1}=0$. From the sequence

$$
\begin{gathered}
0 \rightarrow S^{n-1} I^{*}(-2 n-1) \rightarrow S^{n-2} I^{*} \otimes K(-2 n) \rightarrow \ldots \\
\quad \ldots \rightarrow \wedge^{n-1} K(-n) \rightarrow \wedge^{n-1} E(-n) \rightarrow 0,
\end{gathered}
$$

in order to prove $H^{n}\left(\wedge^{n-1} E(-n)\right)=0$, we only need to see that

$$
H^{n+i}\left(\wedge^{n-1-i} K(-n-i)\right)=0 \quad \text { for } i=0, \ldots, n,
$$


which follows by using the exact sequence (3) exactly as above.

Now, we can state and prove the main result of this section.

Theorem 3.4. $M I_{\mathbb{P} 2 n+1}(k)$ is affine.

Proof. By Theorem 3.3 we get that $\mathcal{Q} \backslash N=\mathcal{Q}^{0}=\mathcal{Q} \backslash\{D=0\}$ is affine. It follows that $M I_{\mathbb{P}^{2 n+1}}(k)$ is affine too, because it is the quotient of an affine variety by a reductive group; see, e.g., [PV89], section 4.4.

As a consequence we deduce

Corollary 3.5. $M I_{\mathbb{P}^{2 n+1}}(k)$ does not contain any complete subvariety of positive dimension.

Proof. This follows from the fact that a quasi-affine complete variety is a finite set.

Remark 3.6. The invariant $D$ is meaningful even in the case $n=0$. In this case it corresponds to the usual determinant of the map $\mathbb{C}^{2 k} \rightarrow \mathbb{C}^{2} \otimes \mathbb{C}^{k}$. For example, for $n=0$ and $k=2$ the degenerate $2 \times 2 \times 4$ matrices fill a variety of codimension 2 and degree $12([\mathrm{BS}])$ in $\mathbb{P}^{15}$ whose ideal is generated by one quartic (which is our invariant $D$ ), 10 sextics and one octic. We remark that the case $2 \times 2 \times 3$ is of boundary format. The case $2 \times 2 \times 5$ is interesting. Here degenerate matrices fill a variety of codimension 3 and degree 20 , and its ideal is generated (at least) by 5 quartics, 50 sextics and 12 octics. The 5 quartics define a variety of codimension 2 and degree 10. Hence, in this case no analog of the invariant $D$ can exist.

\section{INSTANTON BUNDLES With STRUCTURE GROUP $G L(2 n)$}

Definition 4.1. A $k$-instanton bundle $E$ on $\mathbb{P}^{2 n+1}$ is the cohomology bundle of a monad

$$
K \otimes \mathcal{O}(-1) \stackrel{A}{\rightarrow} W \otimes \mathcal{O} \stackrel{B}{\rightarrow} I \otimes \mathcal{O}(1)
$$

where $W$ is a complex vector space of dimension $2 n+2 k$ and $I, K$ are complex vector spaces of dimension $k$.

Notice that $E$ is not necessarily symplectic and that this notion is a true generalization of the one above only for $n \geq 2$, because all rank 2 bundles on $\mathbb{P}^{3}$ with $c_{1}=0$ are symplectic.

Let $(A, B) \in \operatorname{Hom}\left(K \otimes V^{*}, W\right) \times H o m(W, I \otimes V)$ defining $E$. The monad condition is now equivalent to the fact that the matrices $A$ and $B$ are both nondegenerate and $B \cdot A=0$.

$\operatorname{Hom}\left(K \otimes V^{*}, W\right) \times \operatorname{Hom}(W, I \otimes V)$ contains the subvariety $\mathcal{P}$ given by pairs of matrices $(A, B)$ for which the sequence (5) is a complex, that is, such that $B \cdot A=0$. $G L(I) \times G L(K) \times G L(W)$ acts on $\mathcal{P}$ by $(a, b, c) \cdot(A, B)=\left(c A b, a B c^{-1}\right)$.

Arguing, as in the previous section, we can see that there is a natural one-to-one correspondence between

i) isomorphism classes of instanton bundles, and

ii) orbits of $G L(I) \times G L(K) \times G L(W)$ on the open subvariety $\mathcal{P}^{0}$ of $\mathcal{P}$ given by pairs of nondegenerate matrices.

Moreover, as in the second section and using Theorem 4.4, if we denote by $H$ the quotient of $G L(I) \times G L(K) \times G L(W)$ by $\left(\lambda \cdot i d, \lambda^{-1} \cdot i d, \lambda \cdot i d\right)$, then $H$ acts 
freely on $\mathcal{P}^{0}$. In particular, all points of $\mathcal{P}^{0}$ are stable for the action of $G L(I) \times$ $G L(K) \times G L(W)$.

Definition 4.2. The GIT-quotient $\mathcal{P}^{0} / G L(I) \times G L(K) \times G L(W)$ is denoted by $I_{\mathbb{P}^{2 n+1}}(k)$ and is called the moduli space of $k$-instanton bundles on $\mathbb{P}^{2 n+1}$. It is a geometric quotient.

$I_{\mathbb{P}^{2 n+1}}(k)$ coincides for $n=1,2$ with the open subset $\mathcal{I}_{\mathbb{P}^{2 n+1}}(k)$ of the Maruyama scheme of stable bundles on $\mathbb{P}^{2 n+1}$ of rank $2 n$ and Chern polynomial $\frac{1}{\left(1-t^{2}\right)^{k}}$, which are instanton bundles. For $n \geq 3$ we can say that $\mathcal{I}_{\mathbb{P}^{2 n+1}}(k)$ is an open subset of $I_{\mathbb{P}^{2 n+1}}(k)$. We remark that $M I_{\mathbb{P}^{3}}(k)=I_{\mathbb{P}^{3}}(k)$. $\mathcal{I}_{\mathbb{P}^{2 n+1}}(k)$ is known to be singular for $n \geq 2$ and $k \geq 3$ (see [MO97]) and reducible for $n \geq 4$ (see AO00]).

Definition 4.3. Let $(A, B) \in \operatorname{Hom}\left(K \otimes V^{*}, W\right) \times \operatorname{Hom}(W, I \otimes V)$. We define

$$
\tilde{D}(A, B):=\operatorname{det} S(A) \cdot \operatorname{det} R(B)
$$

where det denotes the usual determinant and $S(A), R(B)$ are the morphisms

$$
\begin{aligned}
& S(A): S^{n+1} K \otimes V^{*} \rightarrow S^{n} K \otimes W, \\
& R(B): S^{n} I \otimes W \rightarrow S^{n+1} I \otimes V,
\end{aligned}
$$

induced by $A$ and $B$ respectively, as in Definition 3.1.

Theorem 4.4. If $(A, B)$ defines an instanton (that is, $(A, B)$ belongs to $\left.\mathcal{P}^{0}\right)$, then $\tilde{D}(A, B) \neq 0$.

Proof. First, we will see that $\operatorname{det} S(A) \neq 0$. From (5) we get the exact sequence

$$
0 \rightarrow K \otimes \mathcal{O}(-1) \rightarrow W \otimes \mathcal{O} \rightarrow Q \rightarrow 0 .
$$

The $(n+1)$-th wedge power twisted by $\mathcal{O}(-n-2)$ gives the exact sequence

$$
\begin{aligned}
0 \rightarrow & S^{n+1} K \otimes \mathcal{O}(-2 n-3) \rightarrow S^{n} K \otimes W \otimes \mathcal{O}(-2 n-2) \rightarrow \ldots \\
& \ldots \rightarrow \wedge^{n+1} W \otimes \mathcal{O}(-n-2) \rightarrow \wedge^{n+1} Q(-n-2) \rightarrow 0
\end{aligned}
$$

where the $H^{2 n+1}$ of the first morphism corresponds to $S(A)$. Hence, taking cohomology, it is enough to prove

$$
H^{n}\left(\wedge^{n+1} Q(-n-2)\right)=0 .
$$

This is shown by considering the $(n+1)$-wedge sequence of the exact sequence

$$
0 \rightarrow E \rightarrow Q \rightarrow I \otimes \mathcal{O}(1) \rightarrow 0
$$

and arguing as in the proof of Theorem 3.3 .

In order to prove $\operatorname{det} R(B) \neq 0$, we proceed exactly as in Theorem 3.3 and we leave the details to the reader.

Theorem 4.5. $I_{\mathbb{P}^{2 n+1}}(k)$ is affine.

Proof. First, notice that given $(A, B) \in \mathcal{P}$, if $A$ or $B$ is degenerate, then $\operatorname{det} S(A)$. $\operatorname{det} R(B)=0$. Hence, by Theorem 4.4 we get that $\mathcal{P}^{0}=\mathcal{P} \backslash\{\tilde{D}=0\}$ is affine. Therefore, by [PV89] section 4.4, $I_{\mathbb{P}^{2 n+1}}(k)$ is affine also.

As a by-product of Theorem 4.5, we deduce

Corollary 4.6. $I_{\mathbb{P}^{2 n+1}}(k)$ does not contain any complete subvariety of positive dimension. 


\section{REFERENCES}

[AO94] V. Ancona and G. Ottaviani. Stability of special instanton bundles on $\mathbb{P}^{2 n+1}$. Trans. Amer. Math. Soc., 341, (1994), 677-693. MR 94d:14017

[AO99] V. Ancona and G. Ottaviani. Unstable hyperplanes for Steiner bundles and multidimensional matrices. Advances in Geometry, 1, (2001), 165-192.

[AO00] V. Ancona and G. Ottaviani. On the irreducible components of the moduli space of instanton bundles on $\mathbb{P}^{5}$. Geometry Seminars 1998-1999 (S. Coen, ed.), 95-100, Bologna 2000. MR 2001h:14055

[ADHM78] M. F. Atiyah, V. G. Drinfeld, N. J. Hitchin, and Yu. I. Manin. Construction of instantons, Phys Lett., A65, (1978), 185 -187. MR 82g:81049

[BH78] W. Barth and K. Hulek. Monads and moduli of vector bundles. Manuscripta Math., 25, (1978), 323 -347. MR 80f:14005

[BS] D. Bayer and M. Stillman. Macaulay, a computer algebra system for algebraic geometry (http://www.math.columbia.edu/ bayer/Macaulay.html).

[GKZ94] I. M. Gelfand, M. M. Kapranov, and A. V. Zelevinsky. Discriminants, Resultants and Multidimensional Determinants, Birkhäuser, Boston, 1994. MR 95e:14045

[HH86] A. Hirschowitz and K. Hulek. Complete families of stable vector bundles over $\mathbb{P}^{2}$. Complex analysis and algebraic geometry, Proc. Conf. Göttingen 1985, Lect. Notes Math., 1194, 1986, 19-40. MR 87j:14019

[KO99] P. I. Katsylo and G. Ottaviani. Regularity of the moduli space of instanton bundles $M I_{\mathbb{P} 3}(5) . A G / 9911184,1999$.

[OS86] C. Okonek and H. Spindler. Mathematical instanton bundles on $\mathbb{P}^{2 n+1}$. J. Reine Angew. Math., 364, (1986), 35-50. MR 87e:14016

[MO97] R. M. Miró-Roig and X. Orus-Lacort. On the smoothness of the moduli space of mathematical instanton bundles. Comp. Math., 105, (1997), 109-119. MR 97m:14011

[PV89] V. L. Popov and E. B. Vinberg. Invariant theory. Algebraic geometry. IV: Linear algebraic groups, invariant theory, Encycl. Math. Sci. 55, (1994), 123-278; translation from Itogi Nauki Tekh., Ser. Sovrem. Probl. Mat., Fundam. Napravleniya 55, (1989), 137-309.

[Sal84] S. Salamon. Quaternionic structures and twistor spaces. Global Riemannian Geometry (eds. N. J. Willmore and N. J. Hitchin), Ellis Horwood, London 1984.

[WZ96] J. Weyman and A. Zelevinsky. Singularities of hyperdeterminants. Ann. Inst. Fourier, Grenoble, 46, (1996), 3, 591-644. MR 97m:14050

Departament Algebra i Geometria, Universitat de Barcelona, Gran Via, 585, 08007 BARCELONA, SpAin

E-mail address: costa@mat.ub.es

Dipartimento di Matematica "U. Dini", Università di Firenze, viale Morgagni 67/A, I 50134 Firenze, ItALY

E-mail address: ottavian@math.unifi.it 